10,04

\title{
Быстрые приповерхностные изменения дефектной структуры в кристаллах тетрабората лития во внешнем электрическом поле
}

\author{
() А.Г. Куликов ${ }^{1}$, А.Е. Благов ${ }^{1,2}$, Н.В. Марченков ${ }^{1,2}$, Ю.В. Писаревский ${ }^{1,2}$, М.В. Ковальчук ${ }^{1,2}$ \\ ${ }^{1}$ Институт кристаллограсрии им. А.В. Шубникова Федеральный научно-исследовательский центр \\ „Кристаллографрия и фоотоника“ РАН, \\ Москва, Россия \\ ${ }^{2}$ Национальный исследовательский центр „Курчатовский институт“, \\ Москва, Россия \\ E-mail: ontonic@gmail.com
}

Поступила в Редакцию 14 апреля 2020 г.

В окончательной редакции 11 июля 2020 г.

Принята к публикации 13 июля 2020 г.

\begin{abstract}
Приведены результаты исследования процесса изменения дефектной структуры в приповерхностном слое монокристаллов тетрабората лития $\left(\mathrm{Li}_{2} \mathrm{~B}_{4} \mathrm{O}_{7}\right)$ при воздействии внешнего электрического поля вдоль полярного направления [001]. С помощью метода времяразрешающей (2 ms) рентгеновской дифрактометрии определена динамика изменения параметров (углового положения и интегральной интенсивности) кривых дифракционного отражения рефлексов 004 и 008. Зарегистрировано два типа процессов, вызванных перераспределением заряда, локализованного у поверхности полярного диэлектрика, и миграцией ионов лития, отличающихся по времени реакции на включение внешнего поля, скорости протекания и зависящих от полярности. Измерения проводились при напряжениях, обеспечивающих обратимый характер индуцированных полем эффектов.

Использование двух кратных дифракционных рефлексов с различной глубиной экстинкции рентгеновских лучей позволило получить данные о локализации областей пространственного заряда у анода и катода образца по изменению интегральной интенсивности пиков. Оценка эффективной толщины заряженного приповерхностного слоя дает значение $25 \mu \mathrm{m}$ для ионов лития у катода и около $45 \mu \mathrm{m}$ для вакансий лития у анода.
\end{abstract}

Ключевые слова: миграция носителей заряда, тетраборат лития, времяразрешающая рентгеновская дифрактометрия, внешнее электрическое поле.

DOI: 10.21883/FTT.2020.12.50216.087

\section{1. Введение}

Изучение процессов управления функциональными характеристиками материалов представляет большой интерес для создания новейших систем хранения информации и накопления энергии. Обычно для таких целей используют планарные структуры. Возможность формирования квазидвумерных структур в диэлектриках за счет миграции носителей заряда во внешнем электрическом поле, определяет интерес исследования таких процессов и в случае монокристаллов [1].

Так в монокристаллах титаната стронция $\left(\mathrm{SrTiO}_{3}\right)$ со структурой перовскита был обнаружен и изучен обратимый процесс изменения симметрии, вызванный изменением концентрации кислородных вакансий вблизи поверхности анода образца при воздействии внешнего электрического поля высокой напряженности [2-4]. Данный процесс проявляется в виде уширения кривых дифракционного отражения (КДО). Аналогичные эффекты обнаружены и в кристаллах парателлурита $\left(\alpha-\mathrm{TeO}_{2}\right)$ при приложении электрического поля в направлениях [110] и [100] [5-7]. При этом зарегистрированы два типа процессов. Первый связан с увеличением параметра решетки за счет оттока вакансий и происходит вблизи поверхности анода образца. Второй процесс наблюдался при обеих полярностях прикладываемого электрического напряжения в виде образования медленно релаксирующих доменов с малой угловой разориентацией за счет сильной пьезоэлектрической деформации.

Основные исследования в упомянутых выше работах проводились рентгенодифракционными методами, позволяющими получить информацию о перестройке дефектной структуры, вызванной миграцией носителей заряда. Подобная картина рентгеновской дифракции отражает приповерхностные структурные изменения в кристаллической решетке (искажения за счет дефектов и фазовые переходы), вызванные переносом крупных носителей заряда (ионов и вакансий) в диэлектриках. Титанат стронция и парателлурит обладают невысокой ионной проводимостью при комнатной температуpe с близкими удельными сопротивлениями порядка $\rho=10^{10}-10^{11} \Omega \cdot \mathrm{m}$. Поэтому характерное время (максвелловское время релаксации) миграции носителей заряда и установления равновесия в условиях электрического поля в этих кристаллах составляет сотни секунд, что позволяло изучать динамику дефектной структуры по дифракционным пикам, измеренным посредством традиционного механического $\omega$-сканирования. 
В то же время существует большой класс кристаллических соединений, в которых изменения структуры за счет миграции дефектообразующих носителей заряда (ионов и вакансий) происходят за времена порядка миллисекунд после включения электрического поля [8], и наблюдение динамики эффекта традиционным рентгенодифракционным методом становится невозможным. К таким кристаллам можно отнести тетраборат лития $\left(\mathrm{Li}_{2} \mathrm{~B}_{4} \mathrm{O}_{7}\right)$, в котором обнаружены схожие с вышеописанными эффекты изменения КДО в постоянном внешнем электрическом поле [9].

Недавно были разработаны методики и аппаратные подходы, позволяющие изучать динамику структурных изменений посредством регистрации картины рентгеновской дифракции с милли- и даже микросекундным временным разрешением на синхротронном источнике излучения [10] и лабораторном дифрактометре $[11,12]$. При этом методика основана на периодическом импульсном воздействии внешним электрическим полем, что требует воспроизводимости исследуемых процессов и релаксации образца в исходное состояние в течение некоторого времени после подачи каждого импульса.

В настоящей работе проведено исследование процесса формирования казидвумерных структур, вызванных миграцией носителей заряда (ионов лития) из объема в приповерхностную область в кристалле тетрабората лития во внешнем импульсном электрическом поле, приложенном в направлении полярной оси четвертого порядка. Ввиду сравнительно низкого удельного сопротивления кристалла $\left(\sim 10^{8} \Omega \cdot \mathrm{m}\right)$ и малого расчетного максвелловского времени релаксации измерения проводились с использованием метода времяразрешающей рентгеновской дифрактометрии при импульсном воздействии электрическим полем на образец.

\section{2. Кристаллы тетрабората лития}

\section{1. Свойства}

Тетраборат лития был синтезирован как перспективный материал для использования в инфракрасных передатчиках [13,14]. Впоследствии он нашел применение в газоанализаторах [15], термолюминисцентных дозиметpax ввиду своих пироэлектрических свойств [16,17], а благодаря широкой полосе пропускания - и в нелинейной оптике $[18,19]$. Кроме того, тетраборат лития используется для пьезотехники и акустоэлектроники [20,21], так как обладает сравнимыми по величине с пьезокерамикой пьезоэлектрическими модулями.

Кристаллы тетрабората лития относятся к тетрагональной сингонии, пространственная группа $I 4_{1} c d$, с параметрами решетки $a=b=9.479 \AA, c=10.286 \AA$. Основным структурным элементом тетрабората лития являются бор-кислородные комплексы $\left(\mathrm{B}_{4} \mathrm{O}_{9}\right)$, состоящие из двух плоских тригональных $\left(\mathrm{BO}_{3}\right)$ и двух тетраэдрических групп $\left(\mathrm{BO}_{4}\right)$, в то время как атомы лития локализованы в междоузлиях [22,23]. Основными носителями заряда являются ионы лития [24]. Ионная проводимость $\mathrm{Li}_{2} \mathrm{~B}_{4} \mathrm{O}_{7}$ объясняется вакансиями лития $V_{\mathrm{Li}}^{-}[25]$, так как высокая плотность атомной упаковки и жесткость треугольных и тетраэдрических боркислородных полиэдров препятствуют прямым скачкам ионов лития [26]. Передвижение ионов лития происходит через одномерный канал тетрагональной оси [27]. В направлении полярной оси [001] проводимость почти на 5 порядков выше, чем в перпендикулярных направлениях [28].

Пироэлектрический коэффициент для $\mathrm{Li}_{2} \mathrm{~B}_{4} \mathrm{O}_{7}$ наиболее сильно выражен вдоль направления [001] [29] и при изменении температуры от 50 до $-150^{\circ} \mathrm{C}$ варьируется от $30 \mu \mathrm{C} /\left(\mathrm{m}^{2} \cdot \mathrm{K}\right)$ до $120 \mu \mathrm{C} /\left(\mathrm{m}^{2} \cdot \mathrm{K}\right)[20]$. Спонтанная поляризация в тетраборате лития при комнатной температуре составляет $1.5 \mu \mathrm{C} / \mathrm{cm}^{2}$. При приложении электрического поля вдоль оси четвертого порядка [001] активными являются три компоненты пьезоэлектрического тензора $d_{31}=d_{32}=-2.0 \mathrm{pC} / \mathrm{N}$ и $d_{33}=20.4 \mathrm{pC} / \mathrm{N} \quad$ [30]. Значение диэлектрической проницаемости $\varepsilon=10.0$ [31].

\section{2. Исследуемые образцы}

Исследуемые в настоящей работе кристаллы тетрабората лития были выращены из особо чистого сырья методом Чохральского в платиновых тиглях и атмосфере кислорода. Из полученного кристалла были изготовлены образцы для измерений - монокристаллические плоскопараллельные пластины с линейными размерами $13 \times 9 \times 0.55 \mathrm{~mm}$ и ориентацией нормали к поверхности в направлении [001].

Для удаления нарушенного слоя предварительно отполированные пластины были подвергнуты химическому травлению в 50\%-ом растворе $\mathrm{HNO}_{3}$. На подготовленные поверхности образцов с помощью термического напыления были нанесены сплошные контакты из $\mathrm{Ag}$ толщиной $30 \mathrm{~nm} \mathrm{c}$ подслоем $\mathrm{Cr}$ толщиной $70 \mathrm{~nm}$ для лучшей адгезии с кристаллической поверхностью. Напыление осуществлялось с небольшим (около $0.5 \mathrm{~mm}$ ) отступом от краев образца для предотвращения образования электрической дуги по воздуху.

\section{3. Электрофизические характеристики}

Измерение удельного сопротивления производилось при помощи пикоамперметра (Keithley 6487). Величина электрического тока определялась при разности потенциалов до $200 \mathrm{~V}$ на обкладках образца. Результаты представлены на рис. 1. Линейная зависимость тока от напряжения сохраняется приблизительно до $40 \mathrm{~V}$, а сопротивление составляет $\rho=7.8 \cdot 10^{7} \Omega \cdot \mathrm{m}$. При больших напряжениях наблюдалось падение величины электрического сопротивления от времени. При этом, установившееся значение тока достигалось за несколько сотен секунд. Результирующее значение удельного сопротивления для разности потенциалов на обкладках $200 \mathrm{~V}$ составило $1.7 \cdot 10^{7} \Omega \cdot \mathrm{m}$. Исходя из полученных 


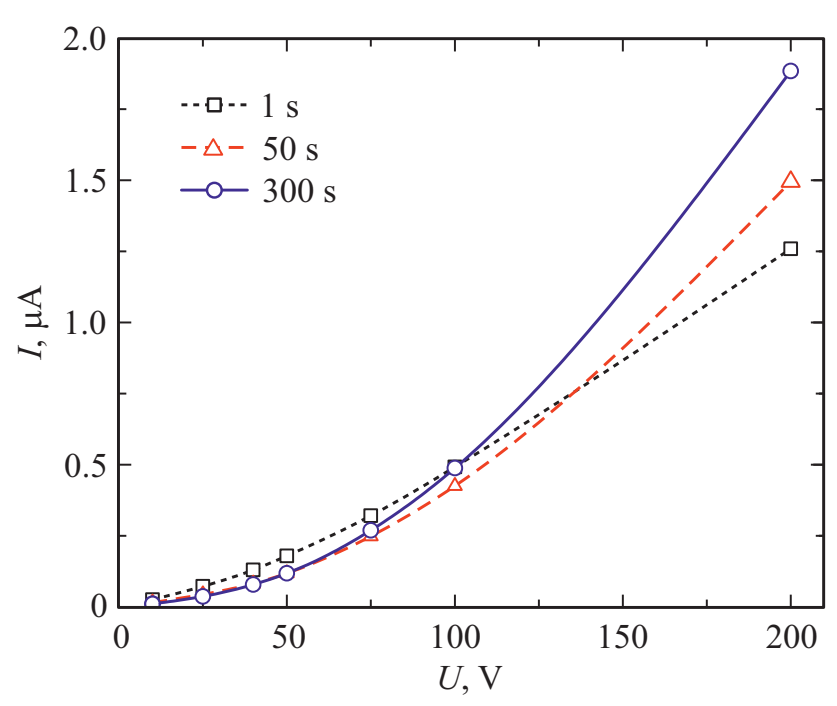

Рис. 1. Зависимость электрического тока от приложенного напряжения при различных временах задержки измерения от начала подачи электрического поля на кристалл.

значений было рассчитано максвелловское время релаксации $\tau_{M}=\varepsilon_{0} \varepsilon \rho$, величина которого составила $3 \mathrm{~ms}$, где $\varepsilon_{0}$ - диэлектрическая постоянная.

\section{3. Методика}

\section{1. Методика рентгенодифракционных измерений с временным разрешением}

Высокоразрешающий двухкристальный метод рентгеновской дифрактометрии позволяет с высокой точностью определять и анализировать параметры КДО выбранного рефлекса. Параметр ширины пика на полувысоте - полуширина (FWHM) и интегральная интенсивность (сумма по углу упругой когерентной дифракционной составляющей и неупругого диффузного рассеяния на дефектах) - является одной из основных характеристик степени совершенства кристалла, угол Брэгга связан с параметром решетки. По вариации данных параметров можно судить об изменениях реальной структуры кристалла [32-34].

Суть времяразрешающей методики двухкристальной рентгеновской дифрактометрии в условиях приложения к образцу внешнего электрического поля высокой напряженности подробно описана в $[11,12]$. КДО исследуемого кристалла измеряется в стандартной двухкристальной геометрии дифракции посредством $\omega$-сканирования образца. Система высокоточной TTL синхронизации на базе генератора электрических импульсов (Tektronix) управляет всем оборудованием, задействованным в эксперименте (рис. 2): многоканальным анализатором интенсивности (ORTEC Easy-MCS), высоковольтным источником постоянного напряжения и гониометром. Для каждого углового положения образца запускается цикл измерения, в процессе которого в определенный момент времени на образец импульсно подается электрическое поле заданной высоковольтным источником (Matsusada) напряженности. Анализатор MCS позволяет разделить дифрагированный образцом сигнал на короткие временные интервалы, длительность которых и задает реальное временное разрешение (кратно $100 \mathrm{~ns}$ ). По завершении эксперимента полученные данные имеют вид набора временных зависимостей интенсивности дифрагированного излучения, измеренных для каждого углового положения образца в условиях идентичных актов внешнего воздействия. Таким образом, по изменению параметров КДО можно отследить состояние кристалла в каждый момент времени от начала цикла.

Важно отметить, что неоспоримым преимуществом применяемой в рамках данной работы методики изучения быстрых процессов в монокристалле является возможность определения динамики всех параметров КДО (положения, интегральной и максимальной интенсивностей, а также полуширины пика). Это выгодно отличает данный метод от метода быстрого измерения интенсивности на склоне кривой качания [35], необходимым условием применимости которого является неизменность формы дифракционного максимума.

\section{2. Экспериментальное оборудование}

Вышеописанная методика времяразрешающей рентгенодифракционной диагностики реализована на базе лабораторного прибора - трехкристального рентгеновского спектрометра (ТРС) [36,37]. Установка (рис. 2) оборудована прецизионным многокружным гониометром с субсекундной точностью углового позиционирования в шаговом режиме сканирования, что позволяет измерять пики КДО в высокоразрешающем режиме для дальнейшей количественной обработки результатов. Источником излучения служила молибденовая рентгеновская трубка с длиной волны $K a_{1}$-линии 0.70932 Ӑи мощностью до $2.5 \mathrm{~kW}$. Монохроматизация пучка осуществлялась за счет дифракционного отражения 220 от высокосовершенного кристалла $\mathrm{Si}$ среза (110) и установленных перед образцом щелей размером $0.2-5 \mathrm{~mm}$. Полуширина собственной КДО для высокосовершенного монокристалла $\mathrm{Si} 220$ составляет 2.1829 arcsec. Для измерения дифрагированного образцом излучения использовался сцинтилляционный детектор (Radicon) с динамическим диапазоном интенсивности $5 \cdot 10^{5} \mathrm{cps}$. Щель перед детектором была раскрыта из соображений накопления достаточной статистики в пике.

\section{3. Параметры рентгенодифракционного эксперимента с временным разрешением}

Измерения проводились при нормальных условиях и термостатировании экспериментального объема. На обкладки образца импульсно подавалось напряжение $500 \mathrm{~V}$, что с учетом толщины кристалла соответствует модулю напряженности электрического поля в его объеме $900 \mathrm{~V} / \mathrm{mm}$. Так как направление [001] является полярным, измерения проводились при двух знаках внешнего 


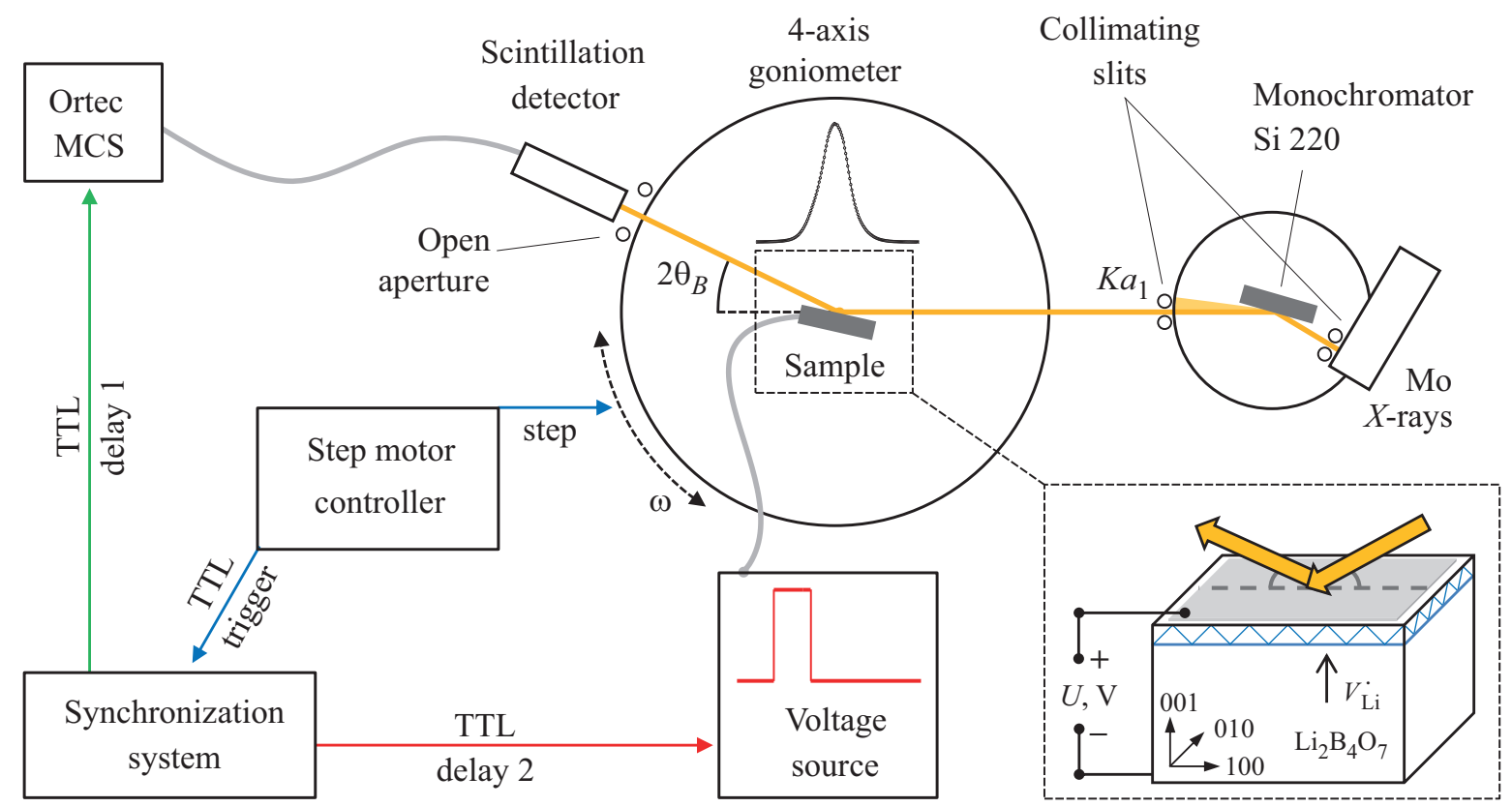

Рис. 2. Схема экспериментальной установки времяразрешающей двухкристальной дифрактометрии с использованием многоканальной системы детектирования.
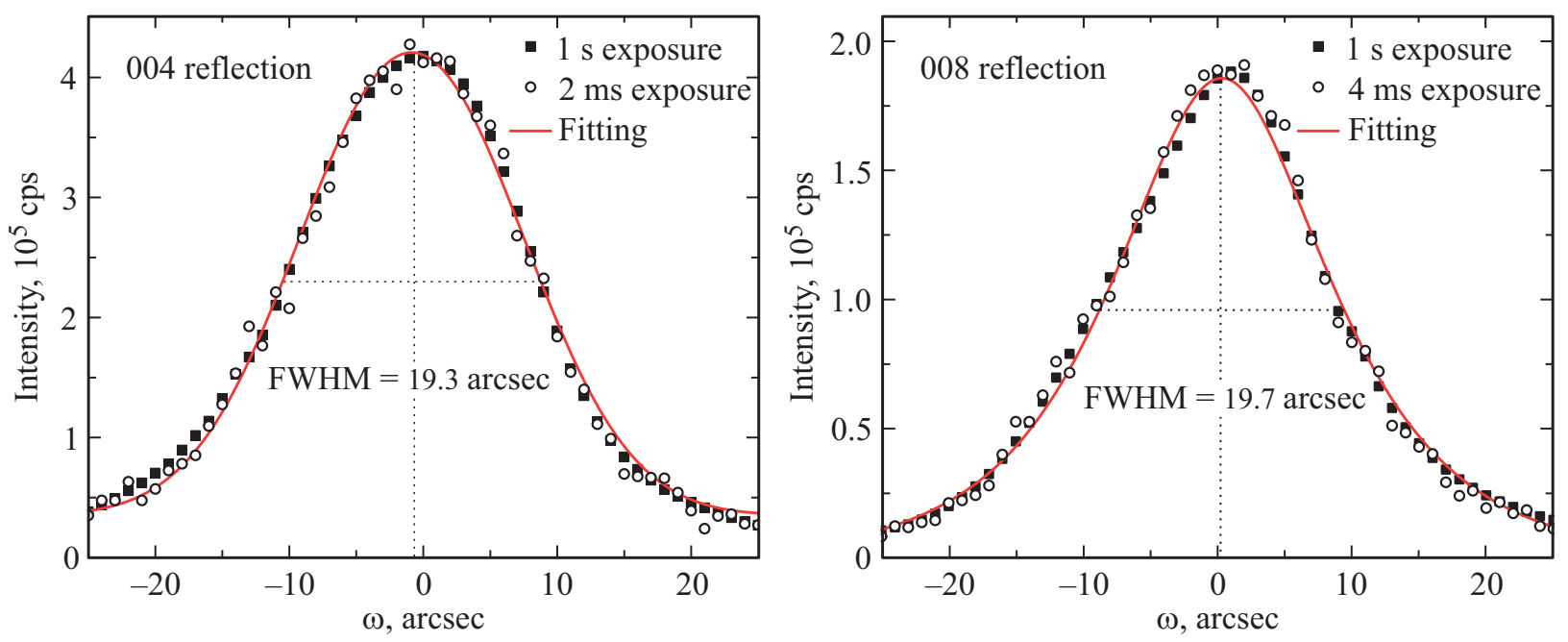

Рис. 3. КДО рефлексов 004 и 008 исследуемого кристалла тетрабората лития, полученные с различным временным разрешением (экспозицией в точке). Линией представлена аппроксимация КДО аналитической функцией псевдо-Войт.

электрического поля, и засвечиваемая рентгеновским пучком поверхность являлась анодом или катодом соответственно. Длительность подаваемого импульса электрического поля составляла $3 \mathrm{~s}$, так как происходящие за это время изменения дефектной структуры являются воспроизводимыми (обратимыми). Между импульсами выжидалось значительно большее время, около $1 \mathrm{~min}$, для полной релаксации кристалла в исходное состояние.

Эксперименты были проведены в геометрии „на отражение“ для двух порядков симметричного отражения: 004 (угол Брэгга $\theta_{B}=7.9 \mathrm{deg}$.) и 008 ( $\theta_{B}=16.0 \mathrm{deg}$.), глубина экстинкции рентгеновских лучей $\left(L_{\text {ext }}\right)$ для которых составляла $L_{e x t}=6.0 \mu \mathrm{m}$ и $L_{e x t}=34.1 \mu \mathrm{m}$ соответственно.

Временное разрешение, определяемое длительностью одного канала MCS, задавалось исходя из предельного соотношения сигнал/шум дифрагированного излучения, что необходимо для удовлетворительной аппроксимации всего массива данных аналитической функцией псевдоВойт в программе обработки, написанной на языке программирования Python. В процессе эксперимента форма профиля пика сохраняется (рис. 3). По результатам обработки были построены временные зависимости параметров аппроксимации КДО: ее углового 


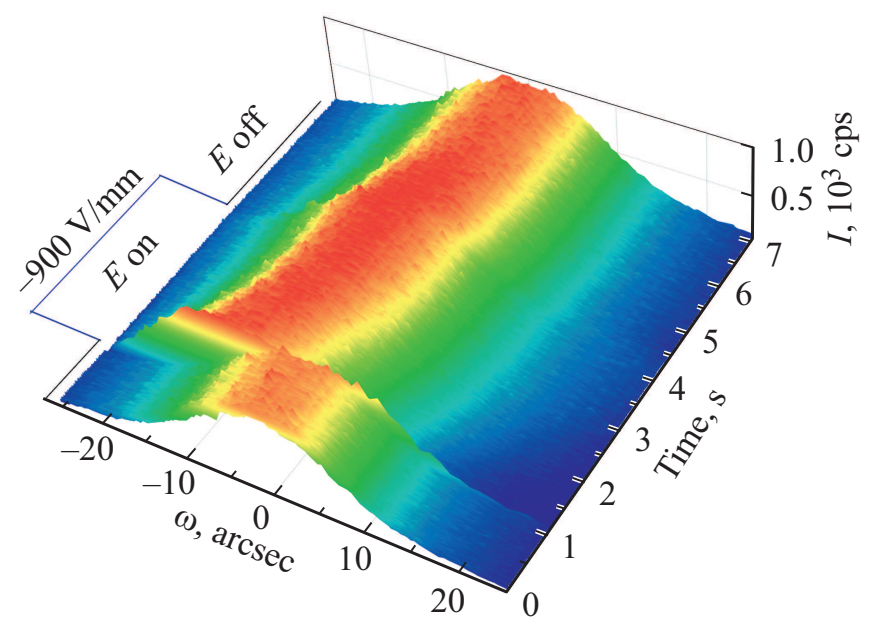

Рис. 4. Эволюция кривых дифракционного отражения (КДО) рентгеновского излучения рефлекса 004 тетрабората лития при воздействии не него внешнего электрического поля напряженностью - $900 \mathrm{~V} / \mathrm{mm}$. Цветом показано распределение интенсивности, соответствующее пику КДО. Временное разрешение составляет $2 \mathrm{~ms}$. Электрическое поле включалось на 1-ой секунде цикла длительностью $60 \mathrm{~s}$, выключалось - на 4-ой. Секундная задержка до подачи поля служит для визуализации стабильности состояния кристалла. Длительность цикла в $60 \mathrm{~s}$ необходима для релаксации кристалла после воздействия. Гониометр перемещается на следующую точку КДО с шагом $0.5 \operatorname{arcsec}$ в конце цикла.

положения и интегральной интенсивности. Для рефлекса 004 было достигнуто временное разрешение $2 \mathrm{~ms}$, а для рефлекса 008, имеющего меньший коэффициент отражения, $-4 \mathrm{~ms}$.

\section{4. Результаты исследования}

На рис. 4 представлена трехмерная картина распределения интенсивности дифрагированного образцом излучения в угловой окрестности точного брэгговского положения рефлекса 004 тетрабората лития при воздействии на него электрическим полем напряженностью $-900 \mathrm{~V} / \mathrm{mm}$. Данная картина соответствует полному массиву данных в координатах время-угол-нтенсивность, получаемому в процессе эксперимента, и отражает динамику изменения КДО с временным разрешением $2 \mathrm{~ms}$.

На рис. 5 представлены результаты обработки экспериментальных данных - угловые зависимости положения дифракционного пика для положительной и отрицательной полярностей соответственно. Цветом выделена область, соответствующая временному интервалу с включенным полем. Видно, что при изменении полярности, меняется также и направление смещения пика. Однако, в начальный момент времени после включения поля смещение дифракционного пика происходит в одном направлении для обеих полярностей. Описанный процесс имеет обратимый характер. При выключении поля наблюдается постепенная релаксация кристалла в исходное состояние.

На рис. 6 представлены графики относительного изменения интегральной интенсивности для двух порядков дифракционного отражения и обеих полярностей. В момент подачи импульса электрического воздействия наблюдается рост интегральной интенсивности пиков с последующей релаксацией в исходное состояние. Амплитуда эффекта имеет наибольшее значение на порядке дифракционного отражения с меньшей глубиной экстинкции и при одной (отрицательной) полярности приложенной разности потенциалов.
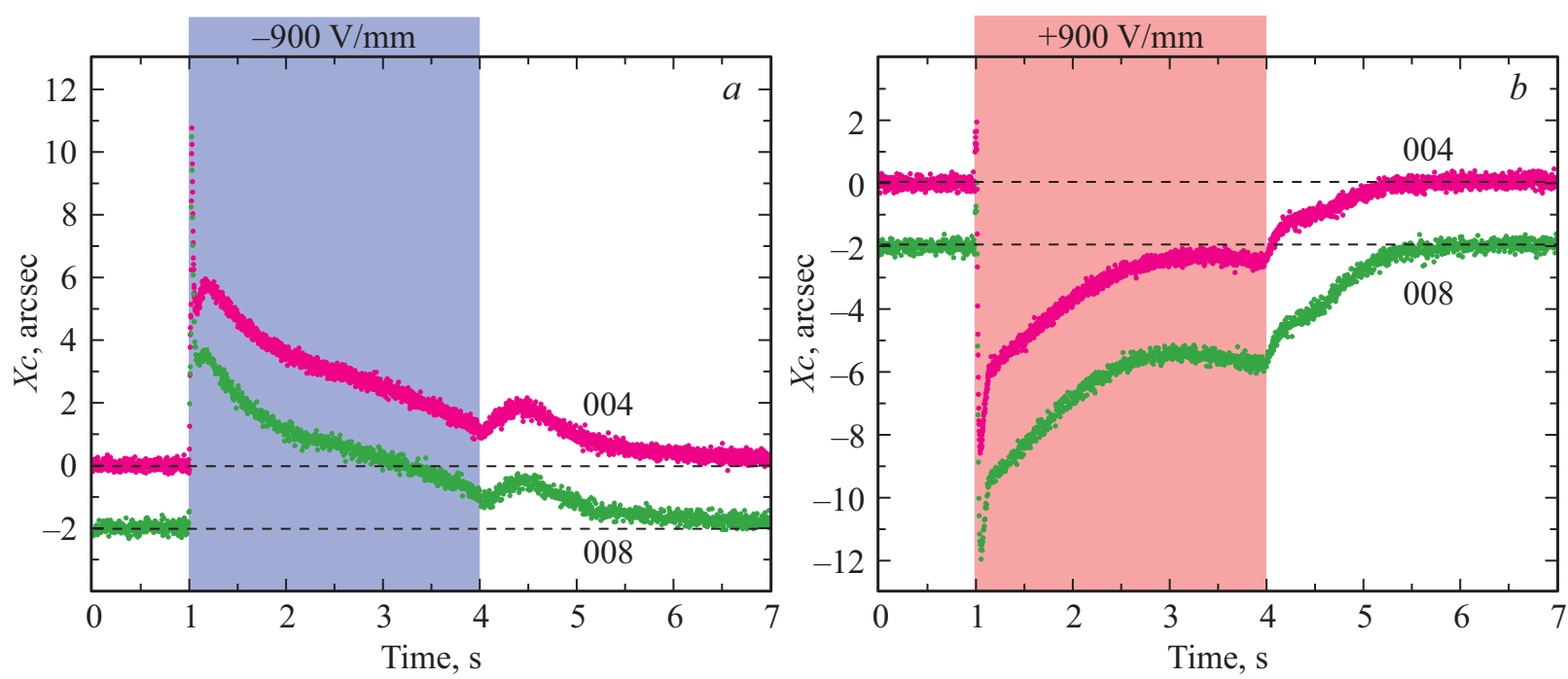

Рис. 5. Временные зависимости положения $X_{c}$ пика КДО по оси $\omega$ для рефлексов $004\left(L_{e x t}=6.0 \mu \mathrm{m}\right)$ и $008\left(L_{e x t}=34.1 \mu \mathrm{m}\right)$ при воздействии внешнего электрического поля напряженностью $-900 \mathrm{~V} / \mathrm{mm}$ отрицательной $(a)$ и положительной $(b)$ полярности на образец. Поле включается на $1 \mathrm{~s}$ и выключается на $4 \mathrm{~s}$ цикла соответственно. Положение максимума для 008 сдвинуто на -2 для наглядности. 

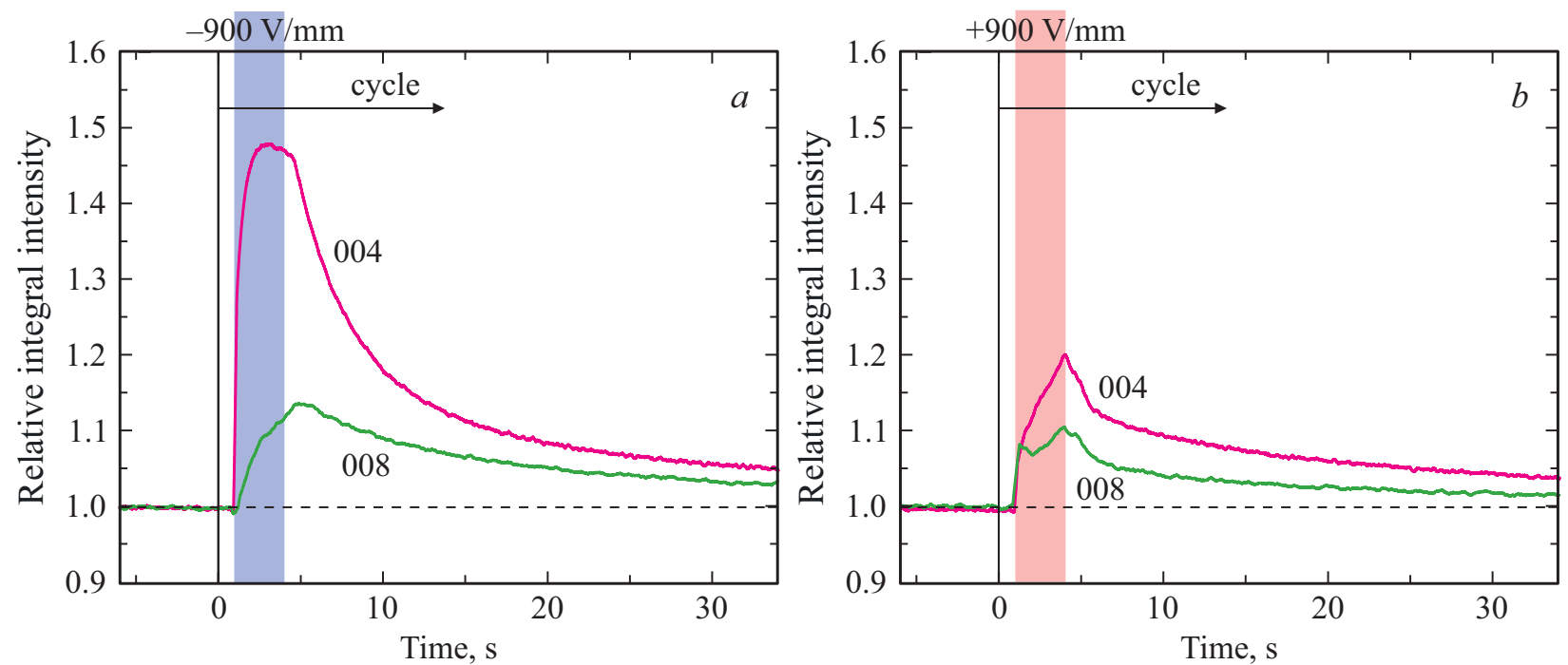

Рис. 6. Временные зависимости изменения интегральной интенсивности в момент подачи поля на образец для двух порядков брэгговского отражения: $004\left(L_{e x t}=6.0 \mu \mathrm{m}\right)$ и $008\left(L_{e x t}=34.1 \mu \mathrm{m}\right)$.

\section{5. Обсуждение результатов}

Зафиксированные обратимые изменения как положения (рис. $5, a, b$ ), так и интегральной интенсивности (рис. $6, a, b)$ дифракционных максимумов при воздействии на кристалл импульсным электрическим полем характерны для процесса движения заряженных дефектов из объема к приповерхностной области кристалла [2-4], динамика которого определяется максвелловским временем. Изменения обеих вышеуказанных параметров КДО несут комплементарную информацию как о деформации кристаллической решетки, так и о дефектной структуре в кристалле.

Наблюдается схожий характер и величина смещения дифракционных максимумов для обоих порядков дифракционного отражения. Поскольку КДО были измерены с открытой щелью перед детектором, изменение углового положения пика на оси $\omega$ вызвано суперпозицией двух изменений - поворотом атомных плоскостей на угол $\Delta \omega_{r o t}$ и вкладом $\Delta \theta_{d}^{h k l}$ за счет изменения межплоскостного расстояния $\Delta d / d$ вдоль оси [001]. Угловой сдвиг экспериментального пика $\Delta \theta_{e x p}^{h k l}$ можно представить как сумму этих двух величин

$$
\Delta \theta_{\text {exp }}^{h k l}=\Delta \theta_{d}^{h k l}+\Delta \omega_{r o t}
$$

Эти два вклада могут быть отделены друг от друга, используя значения углов Брэгга $\theta_{B}^{h k l}$ двух выбранных кратных отражений. В итоге система уравнений будет иметь вид

$$
\begin{gathered}
\frac{\Delta d}{d}=-\frac{\Delta \theta_{d}^{h k l}}{\tan \theta_{B}^{h k l}}=-\frac{\Delta \theta_{e x p}^{000}-\Delta \theta_{\text {exp }}^{004}}{\tan \theta_{B}^{008}-\tan \theta_{B}^{004}} \\
\Delta \omega_{r o t}=\Delta \theta_{\text {exp }}^{004}+\frac{\Delta d}{d} \tan \theta_{B}^{004}=\Delta \theta_{\text {exp }}^{008}+\frac{\Delta d}{d} \tan \theta_{B}^{008}
\end{gathered}
$$

Рассмотрим момент подачи электрического поля более детально. Как видно из рис. 7, $a, b$, основной вклад в смещение пика вносят изгибные деформации. Можно выделить несколько характерных временных промежутков с момента подачи электрического поля. На этих общих для обеих полярностей интервалах $T_{1}, T_{2}, T_{3}$ зависимость деформации кристаллической решетки имеет особенности.

$T_{1}(0-50 \mathrm{~ms})$ - быстрая пьезоэлектрическая деформация кристаллической решетки при включении и стабилизации электрического поля. Для обеих полярностей наблюдаются осцилляции параметров $\Delta d / d$ и $\Delta \omega_{\text {rot }}$, вызванные перераспределением наведенного у поверхности полярного кристалла заряда за максвелловское время. В кристалле возникают разнонаправленные изгибные деформации за счет градиента электрического поля у поверхности (рис. 7,a). К концу данного временного интервала наблюдается стабилизация параметров с разнонаправленной деформацией растяжения/сжатия $\Delta d / d$ (рис. 7, $b$ ).

$T_{2}$ (> $\left.50 \mathrm{~ms}\right)$ - на данном этапе формирование заряженных слоев в кристалле вблизи электродов продолжается уже за счет миграции ионов лития $\mathrm{Li}^{+}$и вакансий лития $V_{\mathrm{Li}}^{-}$из объема. У поверхности с положительным внешним потенциалом (анод) формируется слой $V_{\mathrm{Li}}^{-}$. Наблюдается увеличение параметра решетки за счет сильной пьезоэлектрической деформации (рис. 7,b) с установившимся значением $5 \cdot 10^{-5}$. При этом величина пьезоэлектрической деформации (пьезомодуль $d_{33}$ ) в случае однородного по толщине распределения поля меньше и должна составлять $1.9 \cdot 10^{-5}$ [30]. Для поверхности с отрицательным потенциалом (катод) формируется слой с повышенной концентрацией $\mathrm{Li}^{+}$в кристаллической решетке, параметр которой постепенно растет. Для обеих полярностей также наблюдаются разнонаправленные изгибные деформации (рис. 7,a). 

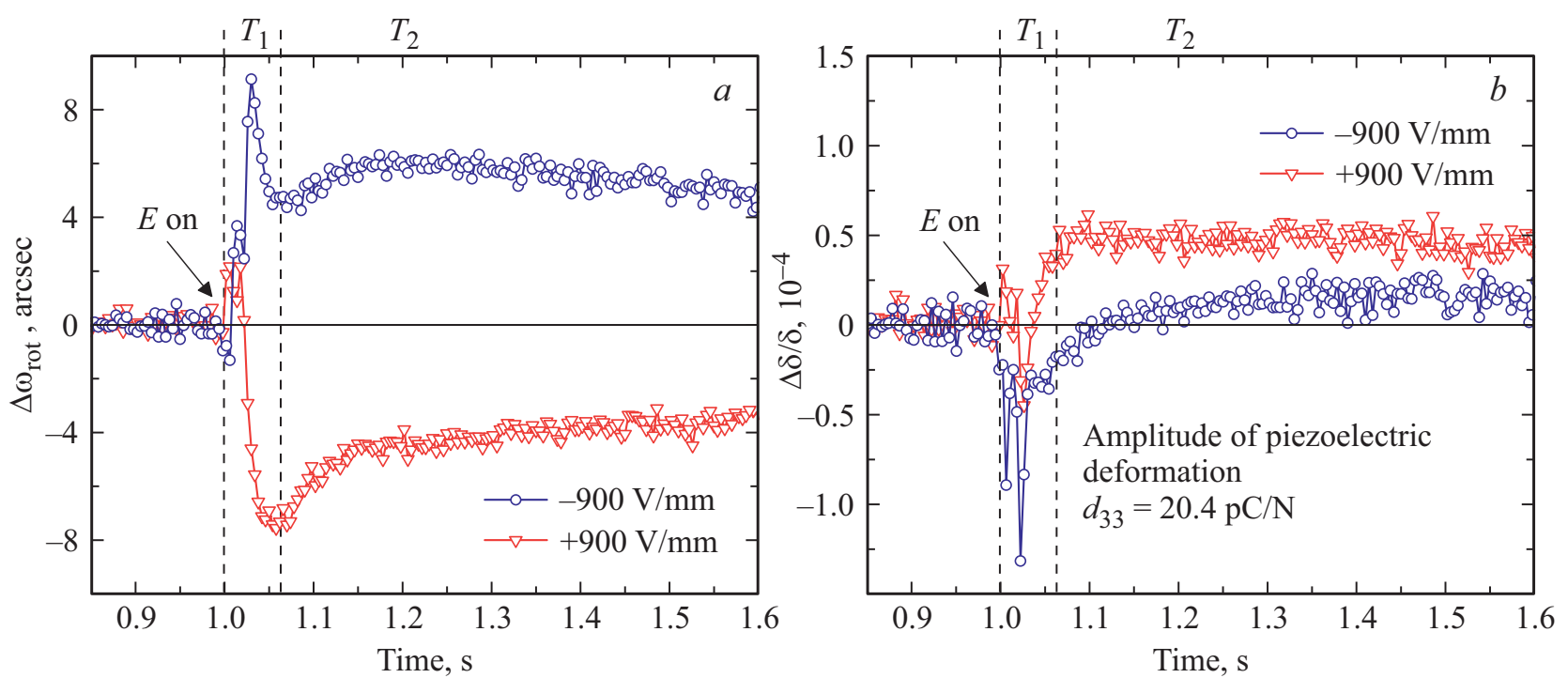

Рис. 7. Графики зависимости угла поворота атомных плоскостей $\Delta \omega_{r o t}(a)$ и изменения параметра кристаллической решетки $\Delta d / d(b)$, рассчитанные по двум кратным порядкам дифракционного отражения 004 и 008 , в начальный момент включения электрического поля для анода (красным) и катода (синим). Положительное значение деформации $\Delta d / d$ соответствует расширению, а отрицательное - сжатию кристаллической решетки.

Далее к концу электрического импульса наблюдается частичное экранирование внешнего поля. После его выключения скопившийся у обкладок заряд постепенно релаксирует за время, не превышающее периода импульсного электрического воздействия.

Энергия активация ионов лития для монокристаллического $\mathrm{Li}_{2} \mathrm{~B}_{4} \mathrm{O}_{7}$ при комнатной температуре равна $0.35 \mathrm{eV}$ [38]. Энергия активации кислородных вакансий в тетраборате лития заметно выше, около $1.65 \mathrm{eV}$, что соответствует меньшей величине проводимости по кислороду и значительно большим характерным временам перемещения кислородных вакансий в кристалле. Приложение импульсного электрического поля (с длительностью импульса $3 \mathrm{~s}$ ) позволяет наблюдать динамику преимущественно одного типа носителей зарядов ионов лития.

Скопление носителей заряда вызывает образование компенсирующего электрического слоя с высокой напряженностью электрического поля в приповерхностном слое кристалла толщиной, сопоставимой с глубиной экстинкции рентгеновского излучения, т.е. около нескольких десятков микрон.

Наиболее отчетливо концентрацию дефектов у поверхности с усреднением по некоторому слою, толщина которого определяется экстинкцией, характеризуют измерения интегральной интенсивности (рис. 6) на различных порядках дифракционного отражения 004 и 008. При этом эффект также чувствителен к полярности. Временна́я зависимость изменения полуширины FWHM пика (не приведена на рисунках) идентична интегральной интенсивности, что подтверждает взаимосвязь данных параметров с динамикой дефектной структуры.

Для отрицательной полярности (у катода) скопление в приповерхностной области большого количества мобильных атомов $\mathrm{Li}^{+}$вызывает сильный, около $50 \%$ для рефлекса 004, рост интегральной интенсивности дифрагированного излучения, что в 3 раза больше, чем для рефлекса 008. Временной характер роста близок к экспоненциальному закону. Для рефлекса 004 процесс занимает характерное время $\tau \approx 370 \mathrm{~ms}$, тогда как для рефлекса 008 с большей в 5.6 раз глубиной экстинкции накопление ионов занимает время $\tau \approx 1520 \mathrm{~ms}$ за счет пропорционально большей емкости данного слоя.

В то же время для положительной полярности, соответствующей аноду, скопление вакансий лития $V_{\mathrm{Li}}^{-}$в приповерхностной области кристалла также вызывает изменение интегральной интенсивности рефлексов (около 10-20\%) за счет оставшихся бор-кислородных комплексов, которые деформируют исходную кристаллическую решетку. Для всех описанных случаев релаксация заряда после выключения поля происходит существенно дольше.

На основании различий в интегральной интенсивности были проведены качественные оценки распределения заряда у поверхности, приведенные на рис. 8, в момент окончания импульса воздействия. Расчеты произведены для электростатического случая [39], при котором распределение электрического потенциала $\varphi$ и наведенной плотности заряда $\rho$ по глубине $z$ подчиняется закону

$$
\begin{gathered}
\varphi(z)=\varphi_{0} e^{-\frac{z}{\lambda},} \\
\Delta \varphi(z)=-\frac{1}{\varepsilon \varepsilon_{0}} \rho(z),
\end{gathered}
$$

где $\varphi_{0}-$ электрический потенциал на поверхности кристалла, $\lambda-$ длина экранирования, $\delta-$ оператор Лапласа.

Оценка эффективной толщины заряженного слоя $\lambda$ для катода дает значение около $25 \mu \mathrm{m}$, что почти в 2 раза 


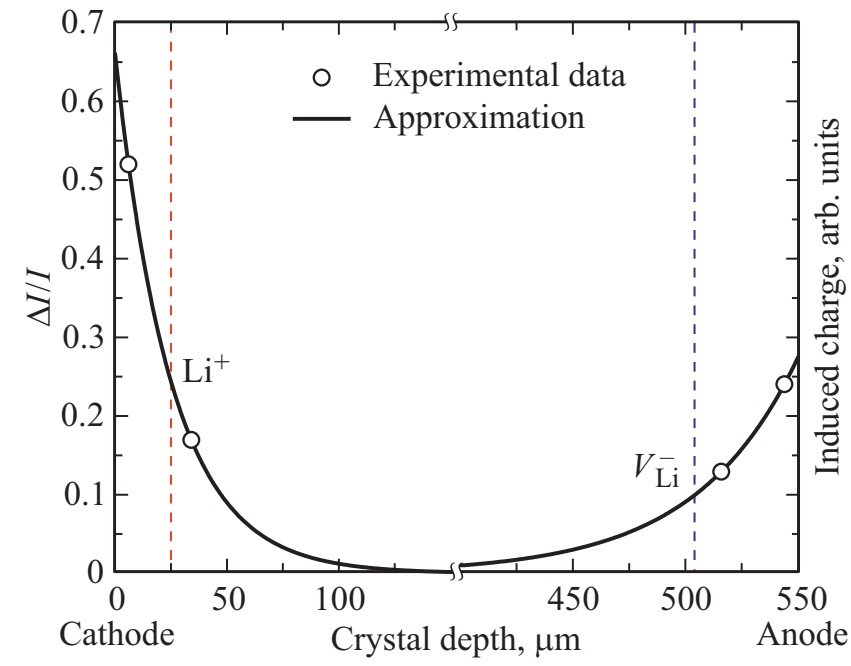

Рис. 8. Изменение интегральной интенсивности $\Delta I / I$ рефлексов 004 и 008 , имеющих различную глубину экстинкции (точки) и соответствующее предполагаемое распределение плотности заряда $\rho$ (сплошная линия) в приповерхностной области кристалла, вызванное миграцией ионов лития. Аппроксимация экспоненциальной функцией выполнена в соответствии с уравнением (5). Штриховыми линиями показана эффективная толщина заряженных слоев.

меньше эффективной толщины слоя для анода (около $45 \mu \mathrm{m})$.

Результаты свидетельствуют о концентрации заряда у поверхности со стороны катода и более размытом его распределении вблизи поверхности кристалла с положительным потенциалом.

Сильные отличия в кинетике интегральной интенсивности КДО в зависимости от порядка дифракционного отражения и полярности подтверждают различный характер формируемых приповерхностных областей заряда в кристалле со стороны катода и анода: большого количества положительно заряженных ионов лития и отрицательно заряженных комплексов $\mathrm{BO}_{3}$ соответственно.

\section{6. Выводы}

С помощью времяразрешающей рентгенодифракционной методики выделено два типа процессов изменения дефектной структуры, различающихся по кинетике протекания, в кристалле тетрабората лития под действием импульсного электрического поля, приложенного вдоль полярной оси. Первый, с характерными временами десятки миллисекунд, вызван перераспределением свободных носителей заряда у поверхности, индуцированных за счет собственной поляризации кристалла. Второй, с временами порядка нескольких секунд, связан с миграцией носителей заряда $\mathrm{Li}^{+}$и $V_{\mathrm{Li}}^{-}$из объема и локализацией их у поверхности. Характерные времена процессов, изучаемых в настоящей работе, определяются высокой ионной проводимостью в тетраборате лития.
C помощью времяразрешающего метода рентгеновской дифрактометрии по изменению интегральной интенсивности дифракционных пиков двух порядков отражения при воздействии полем обеих полярностей определены толщины областей локализации пространственного заряда в приповерхностном слое у обоих электродов образца. Скопление у поверхностей образца носителей заряда разного типа $\left(\mathrm{Li}^{+}\right.$и $\left.V_{\mathrm{Li}}^{-}\right)$для противоположных полярностей прикладываемого электрического поля хорошо согласуется с наблюдаемым различием в характере изменений дефектной структуры.

\section{Благодарности}

Авторы выражают благодарность А.С. Ильину за помощь в проведении электрофизических измерений и И.И. Аткнину за помощь в обработке экспериментальных данных.

\section{Финансирование работы}

Работа выполнена при поддержке Министерства науки и высшего образования в рамках выполнения работ по Государственному заданию ФНИЦ „Кристаллография и фотоника“ РАН в части „выращивания, подготовки кристаллов и обработки экспериментальных данных“ и Российского фонда фундаментальных исследований (грант № 19-52-12029 ННИО_а) в части „разработки времяразрешающей методики и исследования тетрабората лития в условиях воздействия электрического поля“.

\section{Конфликт интересов}

Авторы заявляют, что у них отсутствует конфликт интересов.

\section{Список литературы}

[1] Y. Watanabe, J.G. Bednorz, A. Bietsch, Ch. Gerber, D. Widmer, A. Beck. Appl. Phys. Lett. 78, 3738 (2001).

[2] J. Hanzig, M. Zschornak, F. Hanzig, E. Mehner, H. Stöcker, B. Abendroth, C. Röder, A. Talkenberger, G. Schreiber, D. Rafaja, S. Gemming, D.C. Meyer. Phys. Rev. B 88, 024104 (2013).

[3] J. Hanzig, M. Zschornak, E. Mehner, F. Hanzig, W. Münchgesang, T. Leisegang, H. Stöcker, D.C. Meyer. J. Phys.: Condens. Matter 28, 225001 (2016).

[4] B. Khanbabaee, E. Mehner, C. Richter, J. Hanzig, M. Zschornak, U. Pietsch, H. Stöcker, T. Leisegang, D.C. Meyer, S. Gorfman. Appl. Phys. Lett. 109, 22, 222901 (2016).

[5] М.В. Ковальчук, А.Е. Благов, А.Г. Куликов, Н.В. Марченков, Ю.В. Писаревский. Кристаллография 59, 6, 950 (2014).

[6] А.Г. Куликов, А.Е. Благов, Н.В. Марченков, В.А. Ломонов, А.В. Виноградов, Ю.В. Писаревский, М.В. Ковальчук. Письма в ЖЭТФ 107, 10, 679 (2018).

[7] A.G. Kulikov, A.E. Blagov, A.S. Ilin, N.V. Marchenkov, Yu.V. Pisarevsky, M.V. Kovalchuk. J. Appl. Phys. 127, 065106 (2020). 
[8] А.К. Иванов-Шиц, И.В. Мурин. Ионика твердого тела. Изд-во СПБ ун-та, СПб (2000). Т. 1.616 с.

[9] А.Г. Куликов, Ю.В. Писаревский, А.Е. Благов, Н.В. Марченков, В.А. Ломонов, А.А. Петренко, М.В. Ковальчук. ФTT 61, 4, 671 (2019).

[10] S. Gorfman, O. Schmidt, M. Ziolkowski, M. von Kozierowski, U. Pietsch. J. Appl. Phys. 108, 064911 (2010).

[11] N.V. Marchenkov, A.G. Kulikov, A.A. Petrenko, Yu.V. Pisarevsky, A.E. Blagov. Rev. Sci. Instrum. 89, 095105 (2018).

[12] Н.В. Марченков, А.Г. Куликов, И.И. Аткнин, А.А. Петренко, А.Е. Благов, М.В. Ковальчук. УФН 189, 2, 187 (2019).

[13] J.D. Garrett, M. Natarajan-Iyer, J.E. Greedan. J. Cryst. Growth 41, 225 (1977).

[14] D. Robertson, I. Young. J. Mater. Sci. 17, 1729 (1982).

[15] R. Mohandoss, S. Dhanuskodi, B. Renganathan, D. Sastikumar. Current Appl. Phys. 13, 957 (2013).

[16] I. Ketsman, D. Wooten, J. Xiao, Ya.B. Losovyj, Ya.V. Burak, V. Adamiv, A. Sokolov, J.C. Petrosky, J.W. McClory, P.A. Dowben. Phys. Lett. A 374, 891 (2010).

[17] J.H. Schulman, R.D. Kirk, E.J. West. In: Proceedings of the international conference on luninescence dosimetry. Stanford (June 1965). CONF-650637, Clearinghouse, Springfield. P. 113-118 (1967). OCLC 841357796.

[18] S. Furusawa, O. Chikagawa, S. Tange, T. Ishidate, H. Orihara, Y. Ishibashi, K. Miwa. J. Phys. Soc. Jpn. 60, 2691 (1991).

[19] R. Komatsu, T. Sugawara, K. Sassa, N. Sarukura, Z. Liu, S. Izumida, Y. Segawa, S. Uda, T. Fukuda, K. Yamanouchi. Appl. Phys. Lett. 70, 3492 (1997).

[20] A.S. Bhalla, L.E. Cross, R.W. Whatmore. Jpn. J. Appl. Phys. 24, 727 (1985).

[21] R.W. Whatmore, N.M. Shorrocks, C. O'Hara, F.W. Ainger, I.M. Young. Electron. Lett. 17, 11 (1981).

[22] С.В. Радаев, Л.А. Мурадян, Л.Ф. Малахова, Я.В. Бурак, В.И. Симонов. Кристаллография 34, 6, 1400 (1989).

[23] L. Hanbin, S. Guangqiu, W. Xiaoqing, W. Jingzhi, S. Denzhong. Prog. Cryst. Growth Character 40, 235 (2000).

[24] А.Э. Алиев, Я.В. Бурак, И.Т. Лысейко. Изв. АН СССР. Сер. неорган. материалы 26, 1991 (1990).

[25] C.-S. Kim, D.J. Kim, Y.-H. Hwang, H.K. Kim, J.N. Kim. J. Appl. Phys. 92, 4644 (2002).

[26] И.М. Ризак, В.М. Ризак, Н.Д. Байса, В.С. Биланич, М.В. Богуславский, С.Ю. Стефанович, В.М. Головей. Кристаллография 48, 4, 727 (2003).

[27] M.M. Islam, T. Bredow, C. Minot. Phys. Chem. B 110, 17518 (2006).

[28] C.-S. Kim, Y.H. Hwang, H.K. Kim, J.N. Kim. Phys. Chem. Glass. 44, 166 (2003).

[29] V.T. Adamiv, Y.V. Burak, D.J. Wooten, J. McClory, J. Petrosky, I. Ketsman, J. Xiao, Y.B. Losovyj, P.A. Dowben. Materials 3, 4550 (2010).

[30] И.М. Сильвестрова, П.А. Сенющенков, В.А. Ломонов, Ю.В. Писаревский. ФТТ 31, 10, 311 (1989).

[31] S. Furusawa, S. Tange, Y. Ishibashi, K. Miwa. J. Phys. Soc. Jpn. 59, 7, 2532 (1990).

[32] V. Holy, J. Kubena. Phys. Status Solidi B. 151, 23 (1989).

[33] V.G. Baiyakhtar, M.V. Kovalchuk, Yu.M. Litvinov, V.B. Molodkin, V.V. Nemoshkalenko, S.I. Olikhovskii, E.N. Kislovskii, A.I. Nizkova. Nucl. Instrum. Meth. Phys. Res. A 308, 291 (1991).

[34] M.G. Tsoutsouva, V.A. Oliveira, J. Baruchel, D. Camel, B. Marie, T.A. Lafford. J. Appl. Cryst. 48, 645 (2015).
[35] S. Annaka. J. Appl. Cryst. 10, 354 (1977).

[36] М.В. Ковальчук, Э.К. Ковьев, Ю.М. Козелихин, А.В. Миренский, Ю.Н. Шилин. Приборы и техника эксперимента 1, 194 (1976).

[37] А.Е. Благов, Н.В. Марченков, Ю.В. Писаревский, П.А. Просеков, М.В. Ковальчук. Кристаллография 58, 1, 28 (2013).

[38] L. Bohaty, S. Haussuhl, J. Liebertz. Cryst. Res. Technol. 24, 1159 (1989).

[39] В.Л. Бонч-Бруевич, С.Г. Калашников. Физика полупроводников. Мир, М. (1977). 678 с.

Редактор Ю.Э Китаев 